\title{
Wetting Behavior of Zirconia nanotubes
}

\author{
Swathi Naidu Vakamulla Raghu ${ }^{\text {a }}$, Manuela Sonja Killian* a \\ ${ }^{a}$ Chemistry and Structure of Novel Materials, University of Siegen, Paul-Bonatz-Str. 9-11, 57076 Siegen, Germany
}

In this work, we investigate the wettability of $\mathrm{ZrO}_{2}$ nanotubes (ZrNT) synthesized via electrochemical anodization of Zirconium. The ZrNT surface shows superhydrophilic behavior while the octadecylphosphonic acid $\left(\mathrm{C}_{18} \mathrm{H}_{37} \mathrm{PO}(\mathrm{OH})_{2}\right)$ modified surface shows superhydrophobic behavior. We demonstrate that the wetting properties are independent of $\mathrm{ZrO}_{2}$ nanotube geometry and length.

Electrochemical anodization is a facile technique used to fabricate highly ordered structures in the sub-micron range. Several metals have successfully demonstrated selforganized porous nanostructure growth under appropriate anodization parameters. ${ }^{[1]}$ Fluoride rich electrolytes contributed a significant advancement to the field, enabling the synthesis of high-aspect ratio nanostructured arrays on 'valve metals' such as zirconia. ${ }^{[2],[3],[4]}$ Tuneable surface properties can exploit applications that are surfaceinteraction dependent, such as e.g. catalysis, filtration or coatings. ${ }^{[5],[6],[7]}$ Zirconia is a biocompatible, high band-gap material that has reportedly demonstrated superior surface properties for the enhanced attachment of molecules. ${ }^{[8],[9],[10]}$ It is used in biomedical devices, sensors and more recently for photocatalytic applications. ${ }^{[11 \mid,[12]}$ We recently report the use of $\mathrm{ZrO}_{2}$ nanotubes (ZrNT) based coatings for outdoor use as it is insusceptible to UVexcitation. ${ }^{[13]}$ With this intent, ZrNT surfaces are modified by a monolayer of octadecylphosphonic acid rendering it super-hydrophobic. ${ }^{[14]}$ In the present study, we synthesize $\mathrm{ZrO}_{2}$ nanotubular structures in a single step anodization process and we investigate the influence of nanotubegeometry on the extent of super-hydrophobicity. Zr foils (99.2 \% purity, Goodfellow UK, $0.125 \mathrm{~mm}$ thickness) were ultrasonically cleaned in acetone, methanol and ethanol followed by rinsing in deionized water and dried under a nitrogen stream. Electrochemical anodization was carried out using a high-voltage potentiostat (Jaissle IMP 88-200 PC) in electrochemical cells with a working area of $1 \mathrm{~cm}^{2}$ and larger cross-sections were fabricated via dip-anodization in an electrochemical bath-type setup. In both cases, a platinum counter electrode was used in a two-electrode setup. Anodization was carried out under various conditions to obtain the respective morphologies. 40nm wide-tubes were achieved with ramping the potential at $1 \mathrm{Vs}^{-1}$ from open circuit potential and kept at $50 \mathrm{~V}$ for $2 \mathrm{~h}$ in a glycerol-based electrolyte consisting of $30 \%$ formamide, $2 \mathrm{wt} \% \mathrm{NH}_{4} \mathrm{~F}$ (Merck) and 4wt\% $\mathrm{H}_{2} \mathrm{O}$. Thick-walled 100nm wide tubes (inner wall diameter $\sim 80 \mathrm{~nm}$, wall thickness $20 \mathrm{~nm}$ ) were synthesized at $50 \mathrm{~V}$ for $30 \mathrm{~min}$ without ramping according to the original electrolyte recipe with the addition of $2 w t \%$ distilled water. Thin-walled ZrNTs ( $100 \mathrm{~nm}$ wide, wall thickness $\sim 3 \mathrm{~nm}$ ) were achieved in 30min at a potential of $90 \mathrm{~V}\left(\mathrm{ramp} 1 \mathrm{Vs}^{-1}\right.$ ) using the original electrolyte. The tube length was varied from 3 to $9 \mu \mathrm{m}$ by adjusting the anodization time. Zr compact oxide films were prepared on $\mathrm{Zr}$ foils under a constant potential of $30 \mathrm{~V}$ for 30 min in a $1 \mathrm{M} \mathrm{H}_{2} \mathrm{SO}_{4}$ (Merck) electrolyte. After the electrochemical treatment, the samples were rinsed with ethanol and distilled water and dried with nitrogen. To impart surface hydrophobicity, the samples were placed in solutions containing $10 \mathrm{mM}$ octadecylphosphonic acid (OPA, $\mathrm{C}_{18} \mathrm{H}_{37} \mathrm{PO}(\mathrm{OH})_{2}$ ) in tetrahydrofuran (THF, Roth) for a period of $24 \mathrm{~h}$ at ambient conditions, forming selfassembled monolayers (SAMs). Upon removal, the samples were rinsed in pure THF for 30s and dried under a filtered nitrogen stream. Scanning electron microscopy (Hitachi SEM FE 4800) was employed for the structural and morphological characterization of the anodized zirconia specimens. For chemical characterization, surface modification with OPA was evaluated using a ToF-SIMS V instrument (ION-TOF, Münster). Static water contact angle (WCA) measurements were performed using a Leica Suite Application (DI water droplet volume, 10 $\mu$ ) to determine wetting behavior. Figure.1(a), shows a zirconia substrate with a native oxide film (as received condition), having a hydrophilic WCA of $\left(\sim 30^{\circ}\right)$ owing to surface hydroxyl groups. ${ }^{[15],[16]}$ All porous samples depicted here showed super-hydrophilic behavior as a result of improved oxide quality and enhanced liquid impregnation when

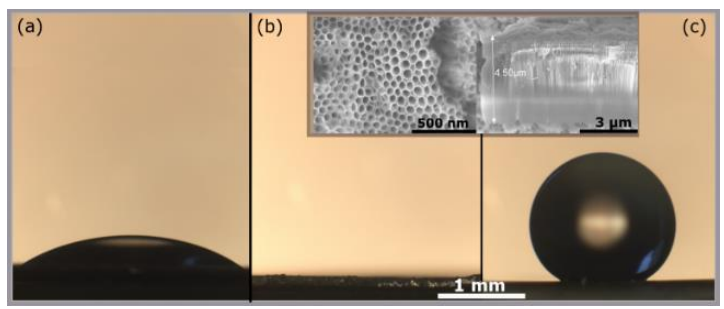

Figure.1: Optical images of water droplet on $\mathrm{ZrO}_{2}$ surface. (a) Native oxide (b) bare ZrNTs (c) ZrNTs after attaching OPA SAM. Inset shows SEM top and cross-section of $\sim 100 \mathrm{~nm}$ ZrNTs.

compared to the native oxide surfaces. The water droplets spread entirely, covering the pores, an example is shown in Figure.1(b). After surface modification with a monolayer of octadecylphosphonic acid, the nanotubes become superhydrophobic as shown in Figure.1(c). To determine the successful attachment of the phosphonic acid to the zirconia substrate, time-of-flight secondary ion mass spectrometry (ToF-SIMS) was performed. The spectra 
represented in Figure.2 confirm the presence of phosphonic acid on the surface of the substrate and are in accordance to reported literature. ${ }^{[10],[17],[18]}$ The quasimolecular signal of OPA $\left(\mathrm{m} / \mathrm{z}=333.29, \mathrm{C}_{18} \mathrm{H}_{38} \mathrm{PO}_{3}{ }^{-}, \mathrm{M}-\mathrm{H}^{-}\right)$was determined as characteristic fragment for the OPA molecule and can be clearly detected on the $\mathrm{ZrO}_{2}$ nanostructures. Figure. 3 shows the WCAs on zirconia test surfaces with different pore configurations; diameters 40nm, thick-walled ( 100nm), thin-walled ( 100nm) nanotubes and compact oxide. Figure.3(a, b, c) reveal that changing the porosity (difference in surface area made up of the solid), did not affect the extent of hydrophobicity, thereby maintaining a nearly constant WCA of $\left(160^{\circ} \pm 1^{\circ}\right)$ on the respective nanotubular surfaces. The only difference appeared when comparing to a flat compact-oxide surface, for which a WCA of $120^{\circ}$ (Figure.3(d)) was recorded. Table.1 summarizes the influence of these pore geometries on WCAs. In Figure.4, WCA measurements were performed on OPA modified ZrNT of varying lengths to evaluate the influence of tube length on the wettability, yielding no statistical changes in dependence of tube length. A zirconia substrate with a native-oxide film as shown in Figure.1(a) shows a hydrophilic WCA. However, when anodized, the nanotubular surfaces experience an enhanced wetting behavior and form super-hydrophilic surfaces. A uniform nanoporous array is a homogenous surface with an increased roughness factor when compared to a 'flat' surface and hence in Figure.1(b), the transition from
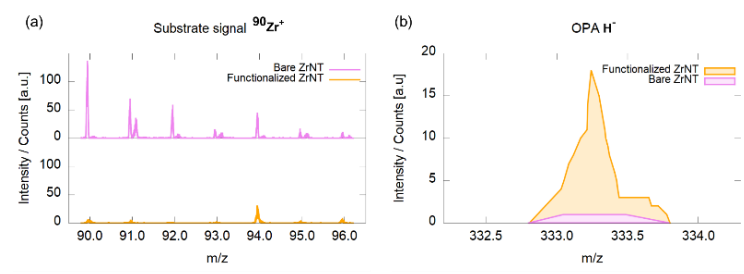

Figure.2: ToF-SIMS spectra of ZrNTs before and after coverage with octadecylphosphonic acid SAMs; (a) $\mathrm{Zr}^{+}$isotopic pattern; (b) OPA-H molecular signal.

hydrophilic to super-hydrophilic state is well accommodated by the Wenzel approach.[19] This phenomenon is represented as an enhanced wetting process that occurs as a result of liquid-solid interaction in the absence of air resistance. After successful surface modification with OPA (Figure.2), the methyl-terminated SAM surface is strongly water-repellent. This results in some cases where the water droplets continue to bounce of the surface and if they do adhere,
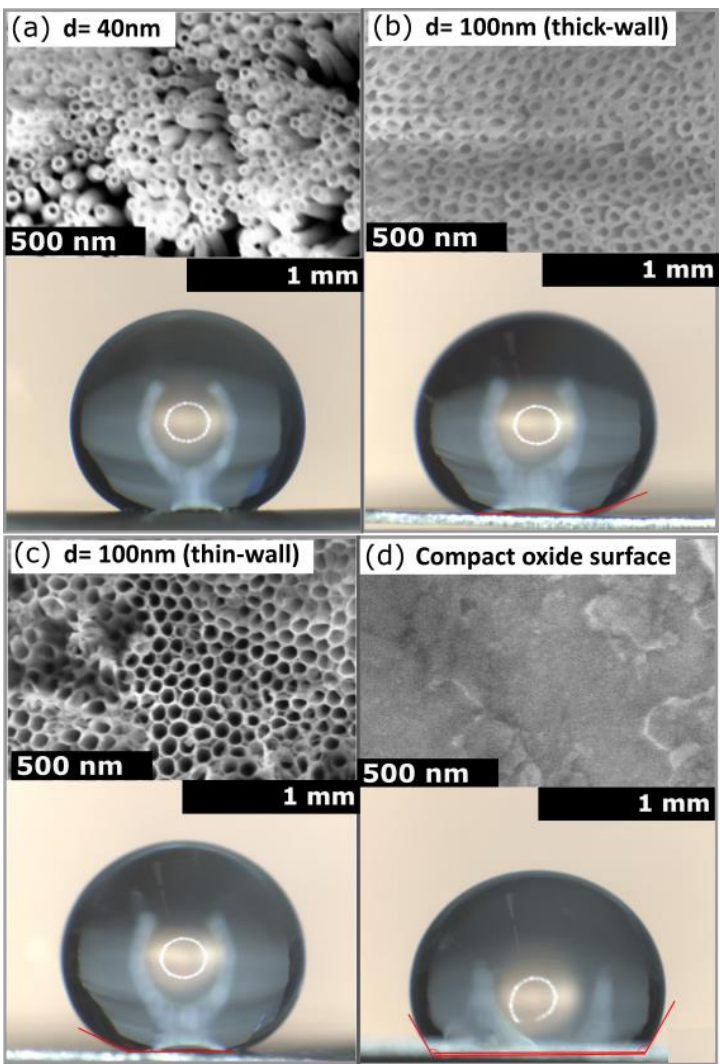

Figure.3: Optical images of water droplets on SAM modified ZrNT structures of different porosity and corresponding SEM images of the surfaces. a) $40 \mathrm{~nm}$ diameter, b) thick walled $100 \mathrm{~nm}$ diameter, c) thin walled $100 \mathrm{~nm}$ diameter and d) compact anodic oxide.

they form high WCAs $\left(\geq 150^{\circ}\right)$. The super-hydrophobic state is stabilized and explained by the Cassie-Baxter model as a result of a greater interaction with trapped air in-between the liquid-solid layers. ${ }^{[20]}$ As represented in Figure.3(a, b, c), the average WCAs measured were $\left(\sim 160^{\circ}\right)$ for the ZrNT surfaces. The WCA values $\left(\theta^{*}\right)$ can be expressed according to the Cassie-Baxter equation

$\cos \left(\theta^{*}\right)=f^{*} \cos (\theta)-(1-f)$

where, $(\theta)$ is the WCA measured on the SAM modified flat zirconia surface, ' $f$ ' is a morphological factor and is defined as the area fraction of solid-liquid contact and $(1-f)$ is the area fraction of air-liquid interface. These surface fractions were calculated from high resolution SEM images as represented in Figure.3, by estimating the ratio between pore walls to the total area respectively and complemented with Image analysis. The morphological factor ' $f$ ' for values represented in Table. 1 was calculated to be $0.047,0.131$,

Table.1: Influence of pore-diameter on WCAs, measured and calculated 
values (according to Cassie-Baxter model and the proposed modification to it)

\begin{tabular}{|c|c|c|c|}
\hline \multirow{2}{*}{$\begin{array}{l}\text { Pore- } \\
\text { diameter } \\
(\mathrm{nm})\end{array}$} & \multirow[b]{2}{*}{ Experiment } & \multicolumn{2}{|c|}{ Water contact angle $\left({ }^{\circ}\right)$} \\
\hline & & $\begin{array}{l}\text { Theoretical } \\
\text { Cassie-Baxter }\end{array}$ & $\begin{array}{c}\text { Theoretical- proposed } \\
\text { Cassie-Baxter }\end{array}$ \\
\hline$\sim 40$ & $159 \pm 2$ & 168 & 167 \\
\hline $\begin{array}{l}\text { Thick-wall } \\
(\sim 100)\end{array}$ & $160 \pm 1$ & 159 & 164 \\
\hline $\begin{array}{l}\text { Thin-wall } \\
(\sim 100)\end{array}$ & $160 \pm 1$ & 167 & 165 \\
\hline Flat surface & $120 \pm 2$ & - & - \\
\hline
\end{tabular}

0.052 for $40 \mathrm{~nm}, 100 \mathrm{~nm}$ thick-walled and thin-walled ZrNTs, respectively.

Recently, it was reported that the wettability of titania nanotubes is dependent on tube diameter and the observations were perfectly supported by Eq.(1) as proposed by the Cassie-Baxter model. ${ }^{[21]}$ For this result to hold true in the case of zirconia, the dimensionless factor ' $f$ in Equation 1 is represented as the ratio between the 'projected area' to the 'total area' where, the projected area $P$ is

$$
P=2 \pi r \varepsilon
$$

where, ' $\varepsilon$ ' denotes the wall thickness, while ' $r$ ' is the radius of the pore. The newly calculated morphological factor ' $f$ ' using Eq. (2) for values represented in Table.1 was calculated to be $0.048,0.079,0.066$ for $40 \mathrm{~nm}, 100 \mathrm{~nm}$ thickwalled and thin-walled ZrNTs, respectively, indicating that the thin-walled NTs are more densely packed than the thick walled. Theoretical values continue to be greater than experimentally measured WCAs for all three morphologies. The experimental values are identical on all substrates, averaging at a value of $\left(\sim 160^{\circ}\right)$. This value is at the physical limit of experimental measurements for low-energy surfaces and, hence, acts as a limiting criterion while quantifying the 'extent' of an already super-hydrophobic surface. ${ }^{[20]}$ This essentially means that the surfaces measured experimentally may indeed have higher WCA values that are in reality closer to the theoretical calculations. Additionally, the proposed modification to the Cassie-Baxter equation accounts for the super-hydrophobic phenomenon exhibited by ZrNTs irrespective of porediameter, because the ' $f$ factor is comparable due to the type of packing exhibited by all three substrates. This implies that for a droplet of fixed volume, the drop-contact line interacts with similar solid fractions and offers a possible mathematical explanation for the superhydrophobicity observed even at smaller pore diameters and is consistent with the constant WCAs when measured experimentally in the case of zirconia whilst still being valid for titania (increasing diameters will increase surface hydrophobicity). ${ }^{[21]}$ Nevertheless, the droplet always remains balanced in the Cassie-Baxter regime. ${ }^{[19],[20]}$ Indeed, there appear a few variations in the theoretically calculated WCA values as compared to the experimental values, which may be attributed to the physical limits of experimental measurement as described earlier. However, both theoretical and experimental values do not deviate significantly and can well be supported and governed by the Wenzel and Cassie-Baxter equations, respectively. Additionally, the WCA of ZrNTs with varying thickness

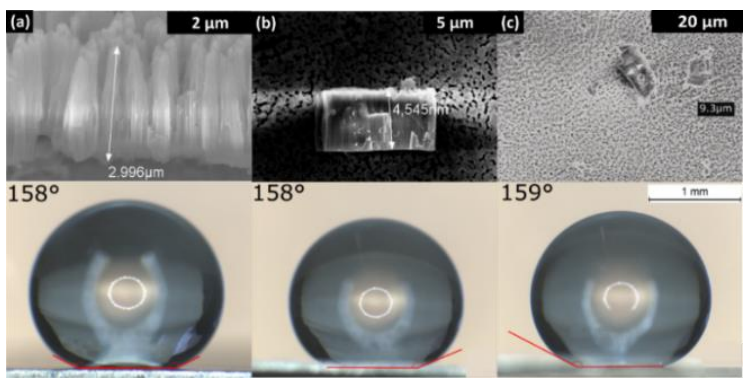

Figure.4: Optical images of water droplets on SAM modified ZrNT structures of different oxide-layer thickness and corresponding SEM images of the cross-sections. length a) $\sim 3 \mu \mathrm{m}$, b) $\sim 4.5 \mu \mathrm{m}$ and c) $\sim 9 \mu \mathrm{m}$.

shows identical values (cf. Figure.4), indicating that the length has a minor effect on surface interactions. In conclusion, the nanoporous $\mathrm{ZrO}_{2}$ layers produced via anodization show a transition from super-hydrophilic to super-hydrophobic surfaces when modified with a (nonfluorinated!) octadecyl phosphonic acid monolayer. The WCAs remain constantly super-hydrophobic $\left(\sim 160^{\circ}\right)$, irrespective of the change in pore diameters and length, thereby following the Cassie-Baxter model. By including the influence of a geometric parameter accommodating the wall thickness, the roughness factor is balanced and continues to remain within the transition threshold of the super-hydrophobic state. Such an observation is of importance as it offers insights into material behavior and is responsible for enhanced degrees of freedom during the fabrication process, such that both simple and complex geometries may still be able to elicit identical surface response. This level of flexibility offers the possibility to work with wider operating parameters involving the anodization process.

There are no conflicts to declare

The authors acknowledge the DFG researcher group FOR 1878 and KI 2169/2-1 for funding. The authors would like to thank Prof. Patrik Schmuki, Dr. Maxime Hubert, Anja Friedrich, Anke Knoop and Ulrike Marten-Jahns for access to lab space, measurements, and discussion. Part of this 
work was performed at the Micro- and Nanoanalytics Facility (MNaF) of the University of Siegen.

\section{References}

(1) Kulkarni, M.; Mazare, A.; Schmuki, P.; Iglic, A. Influence of Anodization Parameters on Morphology of $\mathrm{TiO}_{2}$ Nanostructured Surfaces. Adv. Mater. Lett. 2016. https://doi.org/10.5185/amlett.2016.6156.

Tsuchiya, H.; Macak, J. M.; Sieber, I.; Schmuki, P. Self-Organized High-Aspect-Ratio Nanoporous Zirconium Oxides Prepared by Electrochemical Anodization. Small 2005. https://doi.org/10.1002/smll.200400163.

(3) Amer, A. W.; Mohamed, S. M.; Hafez, A. M.; Alqaradawi, S. Y.; Aljaber, A. S.; Allam, N. K. SelfAssembled Zirconia Nanotube Arrays: Fabrication Mechanism, Energy Consideration and Optical $\begin{array}{llll}\text { Activity. } & \text { RSC } & \text { Adv. } & \end{array}$ https://doi.org/10.1039/c4ra05115g.

(4) Macak, J. M.; Taveira, L. V.; Tsuchiya, H.; Sirotna, K.; Macak, J.; Schmuki, P. Influence of Different Fluoride Containing Electrolytes on the Formation of Self-Organized Titania Nanotubes by $\mathrm{Ti}$ Anodization. J. Electroceramics 2006. https://doi.org/10.1007/s10832-006-3904-0.

(5) Grover, V.; Shukla, R.; Tyagi, A. K. Facile Synthesis of $\mathrm{ZrO}_{2}$ Powders: Control of Morphology. Scr. Mater. 2007. https://doi.org/10.1016/j.scriptamat.2007.06.053

(6) Sanchez, A. G.; Schreiner, W.; Duffó, G.; Ceré, S. Surface Characterization of Anodized Zirconium for Biomedical Applications. Appl. Surf. Sci. 2011, $257 \quad$ (15), 6397-6405. https://doi.org/10.1016/j.apsusc.2011.02.005.

(7) Li, W.; Huang, H.; Li, H.; Zhang, W.; Liu, H. Facile Synthesis of Pure Monoclinic and Tetragonal Zirconia Nanoparticles and Their Phase Effects on the Behavior of Supported Molybdena Catalysts for Methanol-Selective Oxidation. Langmuir 2008. https://doi.org/10.1021/la800370r.

(8) Garcia, J. C.; Scolfaro, L. M. R.; Lino, A. T.; Freire, V. N.; Farias, G. A.; Silva, C. C.; Alves, H. W. L.; Rodrigues, S. C. P.; Da Silva, E. F. Structural, Electronic, and Optical Properties of $\mathrm{ZrO}_{2}$ from $\mathrm{Ab}$ Initio Calculations. J. Appl. Phys. 2006. https://doi.org/10.1063/1.2386967.

(9) Heiroth, S.; Ghisleni, R.; Lippert, T.; Michler, J.; Wokaun, A. Optical and Mechanical Properties of

Amorphous and Crystalline Yttria-Stabilized Zirconia Thin Films Prepared by Pulsed Laser Deposition. Acta Mater. 2011. https://doi.org/10.1016/j.actamat.2010.12.029.

Killian, M. S. Organic Modification of $\mathrm{TiO}_{2}$ and Other Metal Oxides with SAMs and Proteins - a Surface Analytical Investigation. Tech. Fak. 2013.

Piconi, C.; Maccauro, G. Zirconia as a Ceramic Biomaterial. Biomaterials. 1999. https://doi.org/10.1016/S0142-9612(98)00010-6. Yuan, Q.; Liu, Y.; Li, L. Le; Li, Z. X.; Fang, C. J.; Duan, W. T.; Li, X. G.; Yan, C. H. Highly Ordered Mesoporous Titania-Zirconia Photocatalyst for Applications in Degradation of Rhodamine-B and Hydrogen Evolution. Microporous Mesoporous Mater.

2009. https://doi.org/10.1016/j.micromeso.2009.05.00 6.

Raghu, S. N. V.; Chuluunbandi, K.; Killian, M. S. Zirconia nanotube coatings - UV stable superhydrophobic surfaces. Submitted Gao, W.; Dickinson, L.; Grozinger, C.; Morin, F. G.; Reven, L. Self-Assembled Monolayers of Alkylphosphonic Acids on Metal Oxides. Langmuir 1996. https://doi.org/10.1021/la9607621.

Wang, R.; Hashimoto, K.; Fujishima, A.; Chikuni, M.; Kojima, E.; Kitamura, A.; Shimohigoshi, M.; Watanabe, T. Light-Induced Amphiphilic Surfaces [4]. Nature. 1997. https://doi.org/10.1038/41233. Tamura, H.; Mita, K.; Tanaka, A.; Ito, M. Mechanism of Hydroxylation of Metal Oxide Surfaces. J. Colloid Interface Sci. 2001. https://doi.org/10.1006/jcis.2001.7864.

Killian, M. S.; Gnichwitz, J. F.; Hirsch, A.; Schmuki, P.; Kunze, J. ToF-SIMS and XPS Studies of the Adsorption Characteristics of a Zn-Porphyrin on $\mathrm{TiO}_{2}$. Langmuir 2010, 26 (5), 3531-3538. https://doi.org/10.1021/la9032139.

Nie, H. Y. Revealing Different Bonding Modes of Self-Assembled Octadecylphosphonic Acid Monolayers on Oxides by Time-of-Flight Secondary Ion Mass Spectrometry: Silicon vs Aluminum. Anal. Chem. 2010. https://doi.org/10.1021/ac100671q. Wenzel, R. N. Resistance of Solid Surfaces to Wetting by Water. Ind. Eng. Chem. 1936. https://doi.org/10.1021/ie50320a024.

Cassie, A. B. D.; Baxter, S. Wettability of Porous Surfaces. Trans. Faraday Soc. 1944. https://doi.org/10.1039/tf9444000546.

Balaur, E.; Macak, J. M.; Tsuchiya, H.; Schmuki, P. Wetting Behaviour of Layers of $\mathrm{TiO}_{2}$ Nanotubes 
with Different Diameters. J. Mater. Chem. 2005, 15

(42), 4488-4491.

https://doi.org/10.1039/b509672c. 